\title{
Concreto nanoligado a base de sílica fundida para aplicação no riser de unidade de craqueamento catalítico fluido
}

\section{(Nano-bonded fused silica castable for application on fluid catalytic cracking unit riser)}

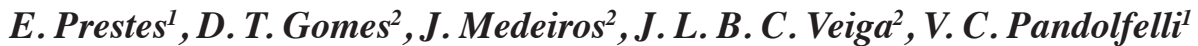 \\ ${ }^{I}$ Departamento de Engenharia de Materiais, Universidade Federal de S. Carlos, Rod. Washington Luiz, $\mathrm{km} 235$, \\ C.P. 676, S. Carlos, SP 13565-905 \\ ${ }^{2}$ Petrobras - CENPES/EB-AB-G-E/EEQ, Rio de Janeiro, RJ \\ epres@uol.com.br,vicpando@ufscar.br
}

\begin{abstract}
Resumo
O agregado de sílica fundida é uma matéria-prima que se mostra adequada para o desenvolvimento de concretos para aplicação em riser de unidade de craqueamento catalítico fluido de petróleo (UFCC). A sua baixa densidade aparente e reduzida condutividade térmica contribuem, respectivamente, para a diminuição do peso da estrutura refratária e maior eficiência térmica do processo. Já o seu coeficiente de expansão linear de $0,55 \times 10^{-6} /{ }^{\circ} \mathrm{C}$, extremamente reduzido, garante uma excelente resistência ao choque térmico. Contudo, para as condições de operação em riser, estas características precisam estar aliadas a um baixo desgaste por erosão. Sendo assim, um concreto nanoligado a base de sílica fundida foi desenvolvido buscando-se a minimização do desgaste por erosão. Duas composições foram testadas combinando-se os agregados de sílica fundida e alumina tabular. Verificou-se que as tensões geradas pelo "mismatch" de expansão térmica entre os agregados resultaram na formação de microtrincas, reduzindo consideravelmente as propriedades termomecânicas do material. Um menor dano foi verificado para a composição onde a alumina tabular foi adicionada somente nas frações mais finas de agregado. Nesta, a incorporação de um aditivo sinterizante favoreceu uma maior densificação em baixa temperatura, possibilitando otimizar a resistência à erosão e ao choque térmico. O valor médio de volume erodido obtido após queima a $815^{\circ} \mathrm{C}$ foi de $4,8 \mathrm{~cm}^{3}$. Em comparação com os $6 \mathrm{~cm}^{3}$ especificados pela indústria petroquímica brasileira para produtos refratários de alta alumina com densidade aparente superior a 2,60 g/ $\mathrm{cm}^{3}$, pode-se afirmar que o resultado obtido foi muito promissor. O concreto nanoligado a base de sílica fundida que foi desenvolvido mostrou ser uma excelente opção para aplicação em riser de UFCC.

Palavras-chave: sílica fundida, mismatch de expansão térmica, concretos nanoligados.
\end{abstract}

\begin{abstract}
Fused silica aggregate shows interesting properties for the development of castables for the riser section of fluid catalytic cracking units (FCCU). Its low apparent density and reduced thermal conductivity can lead to a lightweight refractory structure and a higher thermal efficiency of the process, respectively. The fused silica linear expansion coefficient is very low $\left(0.55 \times 10^{-6} /{ }^{\circ} \mathrm{C}\right)$, allowing an excellent thermal shock resistance. Nevertheless, for the riser environment, these characteristics must be coupled with a low erosion loss. Therefore, a fused silica nano-bonded castable was developed in order to attain a high-erosion resistance. Two compositions containing fused silica and tabular alumina aggregates were evaluated. It was found out that the stress generated by the thermal expansion mismatch between the two sorts of aggregates induced the crack formation, decreasing the material materials' thermomechanical properties. A lower mechanical damage was attained by adding tabular alumina in the finer aggregates grain size. Adding a sintering additive, that induces a higher densification at low temperatures, the erosion and thermal shock resistances were also improved. The mean value of erosion loss obtained after firing at $815^{\circ} \mathrm{C}$ was $4.8 \mathrm{~cm}^{3}$. This result was very promising in comparison with the erosion loss specified by the Brazilian petrochemical industry $\left(6 \mathrm{~cm}^{3}\right)$ for high-alumina refractory products with apparent density higher than $2.60 \mathrm{~g} / \mathrm{cm}^{3}$. The developed fused silica nano-bonded castable seems to be an excellent choice for the riser section of fluid catalytic cracking unit application.
\end{abstract}

Keywords: fused silica, thermal expansion mismatch, nano-bonded castables.

\section{INTRODUÇÃO}

Em determinados tipos de aplicação, as solicitações de serviço dos materiais refratários somente são atendidas por meio da utilização de produtos especialmente projetados. $\mathrm{Na}$ indústria petroquímica esta questão é muito relevante visto que um baixo desempenho pode levar a uma parada de produção, gerando efeitos negativos em relação à rentabilidade do processo. Em unidades de craqueamento catalítico fluido (UFCC) o custo total do material refratário instalado é pequeno em relação ao lucro cessante decorrente de uma parada de produção [1]. Este aspecto deve servir 


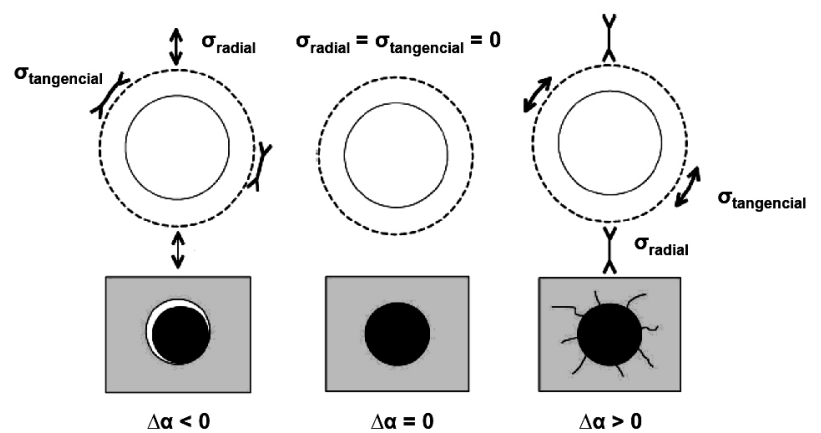

Figura 1: Esquema de uma partícula de agregado envolvida por uma matriz e as tensões que podem ser originadas durante o resfriamento $\left(\Delta \alpha=\alpha_{\text {matriz }}-\alpha_{\text {agregado }}\right)$ [6].

[Figure 1: Schematic representation of internal stresses during the cooling stage of an aggregate particle embedded in a matrix phase $\left.\left(\Delta \alpha=\alpha_{\text {matrix }}-\alpha_{\text {aggregate }}\right)[6].\right]$

como motivação para que os fabricantes apresentem novas soluções. Um requisito fundamental de concretos para aplicação em UFCC é a necessidade de um baixo desgaste por erosão [2]. Contudo, em determinadas sessões desta unidade, tal como o riser, o material também deve possuir uma elevada resistência ao choque térmico e reduzida condutividade térmica, sendo esta última característica muito importante para que a eficiência térmica do processo seja mantida e por servir como uma barreira natural contra o fenômeno de deposição de carbono [2, 3]. Estes requisitos reduzem consideravelmente as opções em termos do tipo de sistema refratário mais adequado. Numa primeira análise, a matéria-prima mais promissora é a sílica fundida, a qual possui uma densidade aparente de $2,20 \mathrm{~g} / \mathrm{cm}^{3}$, condutividade térmica de $1,38 \mathrm{~W} / \mathrm{m} . \mathrm{K}\left(25^{\circ} \mathrm{C}\right)$, coeficiente de expansão de $0,55 \times 10^{-6} /{ }^{\circ} \mathrm{C}$ e temperatura máxima de uso de $1100{ }^{\circ} \mathrm{C}$. A baixa densidade contribui para diminuir o peso da estrutura refratária resultante e o seu coeficiente de expansão, extremamente reduzido, garante uma excelente resistência ao choque térmico. Como a operação em UFCC não ultrapassa os $900{ }^{\circ} \mathrm{C}$ [3], a temperatura máxima de uso se mostra adequada e o risco de que ocorra uma cristalização da sílica amorfa é pequeno. Deste modo, a questão principal fica direcionada para a obtenção de uma elevada resistência à erosão. Uma opção é a combinação do agregado de sílica fundida com outro de maior densidade aparente, o que permitiria uma maior proteção do material por meio do efeito sombra [4]. Neste caso, um adequado arranjo granulométrico é necessário para que as consequiências negativas da diferença de coeficiente de expansão térmica possam ser minimizadas. A literatura mostra que o "mismatch" de expansão pode levar ao trincamento da microestrutura do material devido à geração de elevadas tensões internas oriundas de variações de temperatura, resultando em redução das propriedades termomecânicas $[5,6]$. A Fig. 1 mostra a representação de uma partícula de agregado envolvida por uma matriz e as tensões que podem ocorrer durante o resfriamento considerando-se a diferença de coeficiente de expansão entre as duas fases.

Quando o agregado possui um coeficiente de expansão superior $\left(\Delta \alpha<0 ; \alpha_{\text {matriz }}<\alpha_{\text {agregado }}\right)$, durante o resfriamento, a matriz fica sujeita a tensões de tração radiais, cuja magnitude pode levar ao descolamento da interface matrizagregado. Em uma situação contrária, onde $\left(\Delta \alpha>0 ; \alpha_{\text {matriz }}\right.$ $\left.>\alpha_{\text {agregado }}\right)$, a matriz sofrerá tensões radiais compressivas e tangenciais de tração. Se a matriz não for capaz de acomodar os deslocamentos relativos, o aumento da tensão tangencial levará a ocorrência de um microtrincamento [6]. A erosão também pode ser minimizada pelo aumento da resistência mecânica da matriz do concreto, a qual é a mais propensa ao desgaste por ser uma região que possui menor nível de dureza e maior porosidade [7]. Contudo, em UFCC, as baixas temperaturas de operação dificultam a formação de uma forte ligação cerâmica no material. Uma solução para este problema é a utilização de agentes ligantes nanoestruturados, tal como a sílica e a alumina coloidal, em combinação com aditivos sinterizantes, que favorecem a densificação por meio da formação de uma fase líquida transiente [8-10]. O sinergismo entre a maior reatividade das partículas nanométricas e a capacidade de sinterização em baixa temperatura possibilita a obtenção de materiais antierosivos de elevado desempenho [11]. Este trabalho apresenta o estudo realizado para o desenvolvimento de um concreto a base de sílica fundida para aplicação em riser de UFCC, onde o principal desafio foi a obtenção de uma elevada resistência à erosão.

\section{MATERIAIS E MÉTODOS}

Concretos contendo aluminas tabulares, sílica fundida, alumina calcinada e microssílica foram projetados a partir do modelo de Alfred, com coeficiente de empacotamento igual a 0,26, para aplicação por vibração [12]. A Tabela I apresenta as características das composições refratárias.

Duas formulações a base de sílica fundida contendo frações de alumina tabular SA1 e SA2 foram elaboradas a partir de um sistema de referência de alta alumina (AT). A composição SA1 foi definida por meio da incorporação de 57,12\%-volume de sílica fundida em substituição à AT, sendo que, as frações de alumina tabular intermediárias $[(1 \geq \mathrm{d} \geq 0,5) \mathrm{mm}$ e $(0,2 \geq \mathrm{d}) \mathrm{mm}]$ foram mantidas. $\mathrm{Na}$ composição SA2 o teor de sílica fundida foi aumentado para 68,25\%-volume e, neste caso, somente a fração de alumina tabular $(0,2 \geq d) \mathrm{mm}$ continuou presente na composição. A matriz dos concretos foi composta por alumina calcinada CL370C, sílica fundida e microssílica. Como agente ligante foi utilizada uma suspensão aquosa de sílica coloidal com concentração de $\mathrm{SiO}_{2}$ de 40\%-peso, cujo teor adicionado foi definido com base na obtenção de um índice de fluidez vibrada de $60 \%$. Assim, foram utilizados 5,34\%-volume para o concreto AT e 6,64\%-volume para SA1 e SA2. A sílica coloidal não foi considerada na curva de empacotamento das composições e o teor total de água foi oriundo somente da sua própria suspensão. $\mathrm{O}$ sínter de $\mathrm{MgO}$ foi utilizado no teor de $0,003 \%$-volume, tendo como função favorecer o mecanismo de consolidação por gelificação [13]. O processamento dos materiais foi realizado em um reômetro 
Tabela I - Composição dos concretos refratários.

[Table I - Refractory castable compositions.]

\begin{tabular}{|c|c|c|c|c|c|}
\hline \multicolumn{3}{|c|}{ Matéria-prima (\%-volume) } & \multirow{2}{*}{$\frac{\mathrm{AT}}{82,99}$} & \multirow{2}{*}{$\frac{\text { SA1 }}{-}$} & \multirow{2}{*}{$\frac{\text { SA2 }}{-}$} \\
\hline \multirow{5}{*}{ Agregado } & & $(6 \geq d \geq 0,045) \mathrm{mm}$ & & & \\
\hline & $\begin{array}{l}\text { Alumina tabular } \\
\text { (Almatis, EUA) }\end{array}$ & $(1 \geq \mathrm{d} \geq 0,5) \mathrm{mm}$ & - & 8,72 & - \\
\hline & & $(0,2 \geq \mathrm{d}) \mathrm{mm}$ & - & 14,50 & 9,92 \\
\hline & $\begin{array}{c}\text { Sílica fundida } \\
\text { (CE Minerals, EUA) }\end{array}$ & $(4,75 \geq \mathrm{d} \geq 0,045) \mathrm{mm}$ & - & 57,12 & 68,25 \\
\hline & Alumina tabular & $(0,045 \geq \mathrm{d}) \mathrm{mm}$ & 2,80 & - & - \\
\hline \multirow{3}{*}{ Matriz } & Sílica fundida & $(0,045 \geq \mathrm{d}) \mathrm{mm}$ & - & 9,53 & 12,31 \\
\hline & $\begin{array}{l}\text { Alumina calcinada } \\
\text { (Almatis, EUA) }\end{array}$ & CL370C & 11,25 & 7,70 & 7,24 \\
\hline & $\begin{array}{c}\text { Microssílica } \\
\text { (Elkem, Noruega) }\end{array}$ & MS971D & 2,96 & 2,43 & 2,28 \\
\hline \multirow[t]{2}{*}{ Ligante } & $\begin{array}{l}\text { Sílica coloidal } \\
\text { (Nalco, Brasil) }\end{array}$ & $\begin{array}{c}\text { 00BZL021 } \\
\left(40 \% \text {-peso } \mathrm{SiO}_{2}\right)\end{array}$ & 5,34 & 6,64 & 6,64 \\
\hline & \multicolumn{2}{|c|}{ Teor total de água (\%-volume) } & 4,20 & 5,20 & 5,20 \\
\hline
\end{tabular}

especialmente desenvolvido para concretos refratários [14]. Após a moldagem dos corpos de prova, a cura foi efetuada a $50{ }^{\circ} \mathrm{C}$ por $24 \mathrm{~h}$ em ambiente insaturado (umidade ambiente), seguida de secagem em estufa aquecida a $110^{\circ} \mathrm{C}$. A etapa de queima foi realizada sob uma taxa de aquecimento de $3{ }^{\circ} \mathrm{C} /$ min com patamar de $815^{\circ} \mathrm{C} / 5 \mathrm{~h}$. Os concretos produzidos foram submetidos aos ensaios de densidade e porosidade aparentes, resistência mecânica por compressão diametral, erosimetria a frio e resistência ao choque térmico cíclico. A densidade e a porosidade aparente foram calculadas utilizando-se o método de imersão de Archimedes (ASTM C 830-88). Corpos de prova cilíndricos de $40 \mathrm{~mm}$ x $40 \mathrm{~mm}$ foram utilizados para os ensaios de compressão diametral (ASTM C496-90), sendo que, os testes foram conduzidos em uma máquina universal de ensaios (MTS Systems 810, USA). A resistência à erosão a frio (ASTM C 704) foi determinada para amostras de $115 \mathrm{~mm} \times 115 \mathrm{~mm} \times 25$ $\mathrm{mm}$ que foram ensaiadas em um erosímetro NBR 13185 (Solotest, Brasil). Neste equipamento, partículas erosivas de carbeto de silício são arrastadas por um fluxo de ar e colidem contra a superfície da amostra, sendo esta posicionada a $90^{\circ} \mathrm{em}$ relação ao jato erosivo. No ensaio de resistência ao choque térmico cíclico, amostras de $150 \mathrm{~mm} \times 25 \mathrm{~mm}$ x 25 $\mathrm{mm}$ foram aquecidas $815^{\circ} \mathrm{C}$ e resfriadas ao ar $(\Delta \mathrm{T}=790$ ${ }^{\circ} \mathrm{C}$ ). Este procedimento foi repetido até completar 8 ciclos de ensaio, sendo que, a cada 2 ciclos o efeito do dano por choque térmico foi avaliado pela realização de medidas de módulo elástico, conforme o método de ressonância de barras (ASTM C 1198-91). Com base nos resultados obtidos, a composição mais promissora foi selecionada para incorporação de um aditivo sinterizante, constituído por uma combinação entre Al metálico e um composto a base de boro (em processo de pedido de patente). Por meio da caracterização do concreto aditivado nos ensaios de erosimetria a frio, resistência ao choque térmico cíclico e módulo de ruptura a quente (ASTM C 583-8) foi possível identificar os benefícios decorrentes do uso do aditivo sinterizante. O módulo de ruptura a quente foi determinado para barras prismáticas de $150 \mathrm{~mm} \times 25 \mathrm{~mm} \times 25 \mathrm{~mm}$, previamente queimadas, que foram ensaiadas em uma máquina de flexão a três pontos, HBST (High Bending Strength Tester) 422 (Netzsch, Alemanha), para uma velocidade de carregamento de 12,5 $\mathrm{N} / \mathrm{s}$. Durante o desenvolvimento do concreto nanoligado a base de sílica fundida o ensaio de módulo elástico em função da temperatura foi usado tanto para auxiliar na seleção da composição refratária mais adequada como para verificar o efeito dos aditivos sinterizantes. Como a maioria das transformações (densificação, mudanças de fase, cristalização, etc.) e geração de defeitos (microtrincamento) envolve variações efetivas do módulo elástico, a utilização desta técnica se mostra promissora como ferramenta para avaliar a evolução microestrutural do material [15]. O método de ressonância de barras (ASTM C 1198-91) foi aplicado em corpos de prova dos concretos que, após secagem a $110^{\circ} \mathrm{C}$, foram aquecidos sob uma taxa de $2{ }^{\circ} \mathrm{C} /$ min até $1000^{\circ} \mathrm{C}$ e resfriados até a temperatura ambiente. Obteve-se assim, um perfil correspondente ao primeiro contato com alta temperatura. O comportamento obtido por meio da aplicação de um segundo aquecimento permitiu verificar se as transformações ocorridas no primeiro ciclo térmico foram permanentes.

\section{RESULTADOS E DISCUSSÃO}

$\mathrm{O}$ valor médio de densidade e porosidade aparente dos concretos AT, SA1 e SA2, após queima a $815^{\circ} \mathrm{C} / 5 \mathrm{~h}$, é apresentado na Fig. 2.

A incorporação de sílica fundida na composição de alta alumina foi muito eficiente para o decréscimo da densidade aparente. De acordo com o resultado apresentado para o concreto SA1, contendo 57,12\%-volume de $\mathrm{SiO}_{2}$, o valor médio de $3,19 \mathrm{~g} / \mathrm{cm}^{3}$ foi reduzido para $2,36 \mathrm{~g} / \mathrm{cm}^{3}$. 


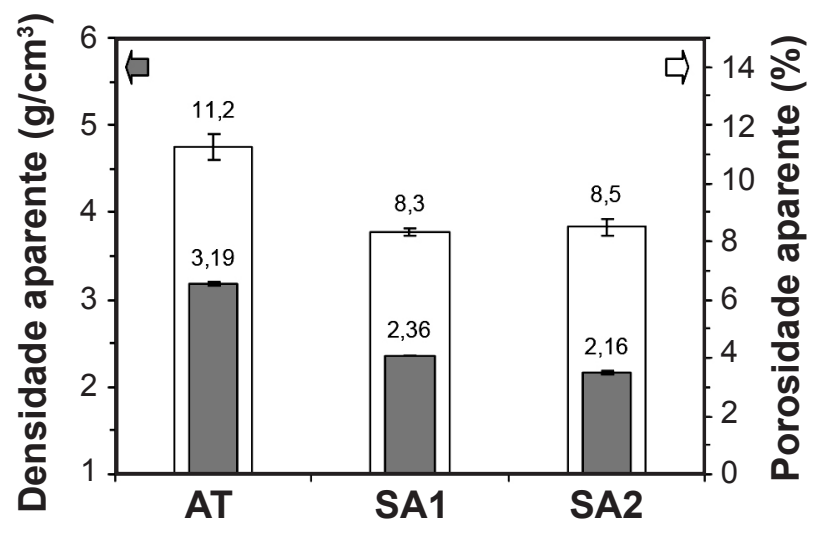

Figura 2: Densidade e porosidade aparente dos concretos AT, SA1 e SA2 após queima a $815^{\circ} \mathrm{C} / 5 \mathrm{~h}$.

[Figure 2: Apparent density and porosity after firing at $815^{\circ} \mathrm{C} / 5 \mathrm{~h}$ for castables AT, SAI and SA2.]

Aumentando-se ainda mais o teor de sílica fundida, para 68,25\%-volume (concreto SA2), foi possível a obtenção de uma densidade inferior a $2,20 \mathrm{~g} / \mathrm{cm}^{3}$. O nível do empacotamento de partículas obtido para estes sistemas contendo sílica fundida foi muito elevado já que para ambos a porosidade aparente foi inferior a do concreto AT, mesmo tendo sido utilizado um maior teor total de água. As composições SA1 e SA2 atenderam o requisito de densidade aparente da indústria petroquímica brasileira para produtos antierosivos aplicados em riser de UFCC, que é de no máximo $2,40 \mathrm{~g} / \mathrm{cm}^{3}$ [16]. Mesmo assim, para poder afirmar que estas são plenamente adequadas é necessário avaliar o seu comportamento em relação ao mismatch de expansão térmica entre a alumina tabular e a sílica fundida, o que pode levar à formação de defeitos microestruturais que resultam em queda da resistência mecânica. Uma importante técnica que pode ser utilizada para este propósito é a do módulo elástico a quente [5,6]. A Fig. 3 apresenta o perfil obtido para os concretos AT, SA1 e SA2 durante o aquecimento até $1000{ }^{\circ} \mathrm{C}$ e, posterior, resfriamento até a temperatura ambiente $\left(1^{\circ}\right.$ ciclo térmico). A alteração de valores representa as transformações ocorridas no material durante o primeiro contato com temperatura elevada, já que as amostras utilizadas não foram previamente queimadas.

O módulo elástico inicial do concreto AT sofreu um aumento durante o aquecimento e se manteve praticamente constante durante todo o resfriamento. Para temperaturas de até $1000{ }^{\circ} \mathrm{C}$ pode-se considerar que isto foi unicamente relacionado a atuação da sílica coloidal utilizada como agente ligante. O comportamento apresentado pelos concretos SA1 e SA2 foi distinto em relação ao material AT. Para estes, os decaimentos observados no valor do módulo elástico são indicativos da formação de defeitos na microestrutura, neste caso, causados pelo mismatch de expansão entre os dois agregados e a matriz dos concretos. Uma análise das tensões que são induzidas pode ser feita com base no trabalho [17] que utiliza um modelo de esferas concêntricas [18], conforme mostra a Fig. 4.

A esfera de raio $a$ representa uma partícula que possui

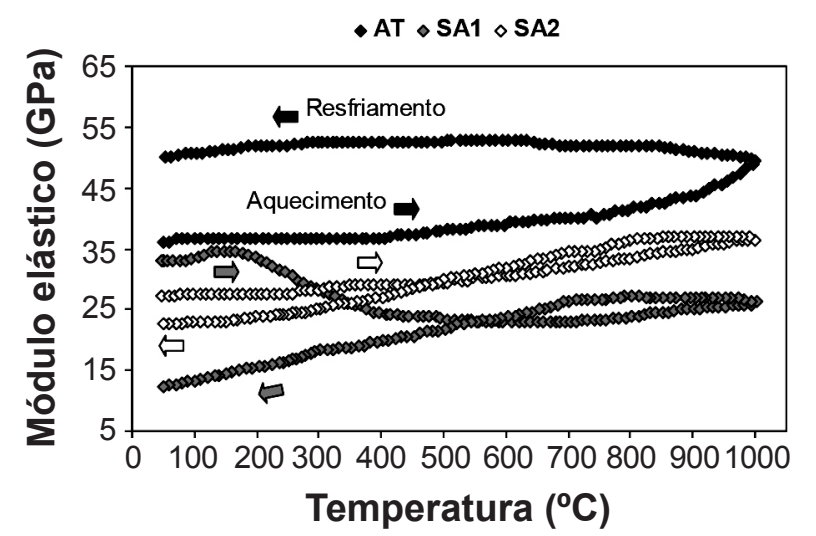

Figura 3: Módulo elástico in situ para o $1^{\circ}$ ciclo térmico dos concretos AT, SA1 e SA2.

[Figure 3: In situ elastic modulus as a function of temperature for AT, SA1 and SA2 castables - first thermal cycle.]

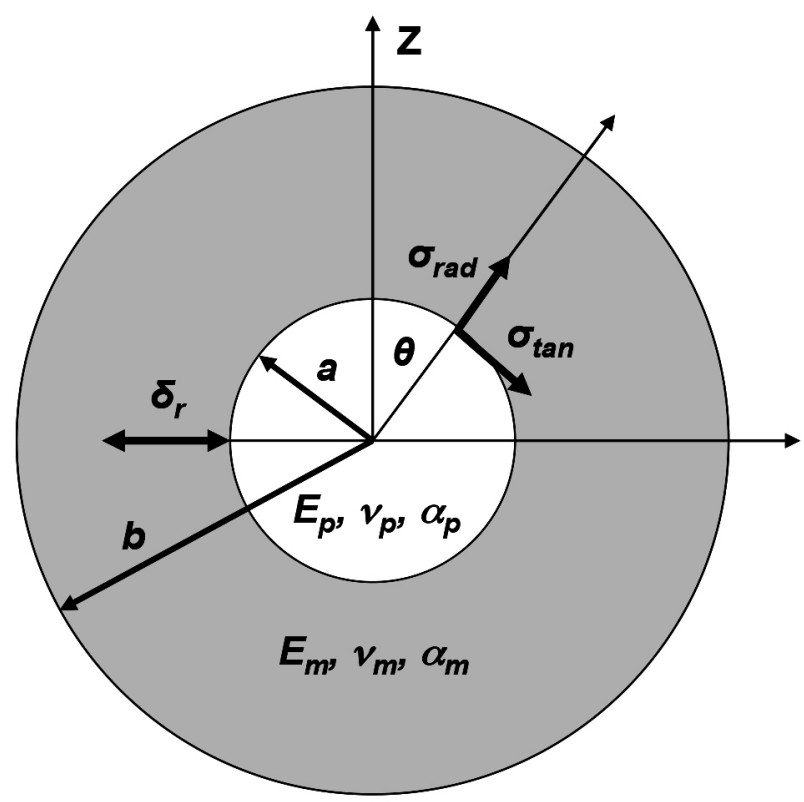

Figura 4: Modelo de esferas concêntricas [17].

[Figure 4: Concentric sphere model [17].]

módulo elástico $(E)$, razão de Poisson $(v)$ e coeficiente de expansão térmica $(\alpha)$ distintos em relação à matriz que a envolve, sendo esta também representada por uma esfera concêntrica de raio $b$. Os subscritos $m$ e $p$ são indicativos de matriz e partícula, respectivamente. As tensões que são geradas na interface matriz-partícula e na própria matriz possuem uma componente radial $\left(\sigma_{r a d}\right)$ e outra tangencial $\left(\sigma_{\tan }\right)$. Estas podem ser calculadas como uma função do tamanho $(a)$ e da fração volumétrica $(x)$ de partículas, conforme mostram as equações A e B [17], onde termo $\delta_{r}$ indica uma distância a partir da interface entre a partícula e a matriz.

$\sigma_{\mathrm{rad}}=\frac{-\left(\alpha_{\mathrm{p}}-\alpha_{\mathrm{m}}\right) \Delta \mathrm{T}}{\frac{2 \mathrm{x}\left(1-2 \mathrm{v}_{\mathrm{m}}\right)+\left(1-\mathrm{v}_{\mathrm{m}}\right)}{2 \mathrm{E}_{\mathrm{m}}(1-\mathrm{x})}+\frac{1-2 \mathrm{v}_{\mathrm{p}}}{\mathrm{E}_{\mathrm{p}}}} \times \frac{\left(\frac{\mathrm{a}}{\mathrm{a}+\delta_{\mathrm{r}}}\right)^{3}-\mathrm{x}}{\mathrm{x}-1}$ 


$$
\sigma_{\tan }=\frac{\left(\alpha_{p}-\alpha_{m}\right) \Delta T}{\frac{2 x\left(1-2 v_{m}\right)+\left(1-v_{m}\right)}{2 E_{m}(1-x)}+\frac{1-2 v_{p}}{E_{p}}} \times \frac{\frac{a^{3}}{x\left(a+\delta_{r}\right)^{3}}+2}{2\left(1-\frac{1}{x}\right)}
$$

A tensão na interface matriz-partícula $\left(\delta_{r}=0\right)$ depende fortemente da fração volumétrica. Se esta for mantida constante, ambas componentes (tangencial e radial) sofrem acréscimo (para uma determinada distância $\delta_{r}$ ) com o aumento do tamanho de partícula. Liu e Winn [17] mostraram que a interação entre duas partículas de mesma composição também é influenciada pelo aumento da fração volumétrica, pois, a consequente redução da distância média entre os agregados faz com que ocorra um aumento do nível de tensão na interface com a matriz. As tensões de tração tangenciais são responsáveis pela formação de trincas radiais e, por outro lado, as trincas circunferenciais são decorrentes de tensões de tração radiais (Fig. 1). De acordo com Green [19], o trincamento radial é geralmente mais prejudicial à resistência mecânica do material do que o circunferencial. A total compreensão do perfil do módulo elástico in-situ dos concretos SA1 e SA2 no primeiro ciclo de queima (Fig. 3) é dificultada pela maior complexidade da microestrutura em relação ao modelo matemático e, também, pelo fato da técnica utilizada não permitir a identificação do tipo de defeito que foi formado. Mesmo assim, foi possível estabelecer uma relação entre os comportamentos observados e as características das duas composições. Na Tabela I, verifica-se que a matriz dos concretos SA1 e SA2, constituída pelas partículas de tamanho inferior a $0,045 \mathrm{~mm}$, foi composta por sílica fundida, microssílica e alumina calcinada. Já os agregados, consistiram de uma combinação entre sílica fundida e alumina tabular. $\mathrm{O}$ coeficiente de expansão das partículas grosseiras de alumina é maior do que o da matriz, tanto para SA1 como para SA2, com isso, pode ocorrer à formação de um trincamento radial durante a etapa de aquecimento. $\mathrm{O}$ contrário, trincamento circunferencial, ocorre para os agregados de sílica fundida. $\mathrm{O}$ fato de nenhuma irregularidade característica ter sido detectada para o concreto SA2 durante o aquecimento, mostra que os deslocamentos relativos foram acomodados pela microestrutura desta composição. Contudo, para SA1 houve uma queda significativa do módulo elástico. Como a principal característica desta formulação foi uma maior presença de agregados de alumina tabular com tamanho de partícula mais elevado, o comportamento observado foi atribuído à ocorrência de trincas radiais, as quais foram favorecidas pelo aumento do nível de tensão na interface partícula-matriz e pela maior fração volumétrica de alumina tabular. Durante o resfriamento, tanto SA1 como SA2 apresentaram uma queda do módulo elástico. Para esta condição, o trincamento radial é vinculado aos agregados de sílica fundida e, o circunferencial, aos de alumina tabular. $\mathrm{O}$ perfil apresentado pelos concretos foi similar, podendo ser resultante da formação dos dois tipos de defeitos. Ao final do primeiro ciclo térmico a queda total do módulo elástico foi de $20 \mathrm{GPa}$, para o concreto SA1, e de $5 \mathrm{GPa}$, para o SA2. Em um $2^{\circ}$ ciclo térmico (Fig. 5), o perfil apresentado foi próximo ao de um loop de histerese, o qual é característico de refratários que sofreram dano microestrutural induzido por mismatch de expansão térmica durante a etapa de queima [20]. Neste caso, o progressivo aumento do módulo elástico durante o aquecimento é atribuído a uma redução gradual do dano inicial (ocorrido no $1^{\circ}$ ciclo térmico) por meio do fechamento de defeitos e trincas. No resfriamento, a partir de uma determinada temperatura, a reversão deste efeito faz com que o módulo elástico diminua até se aproximar do seu valor inicial [5, 6, 21].

No $2^{\circ}$ ciclo uma maior formação de defeitos não foi

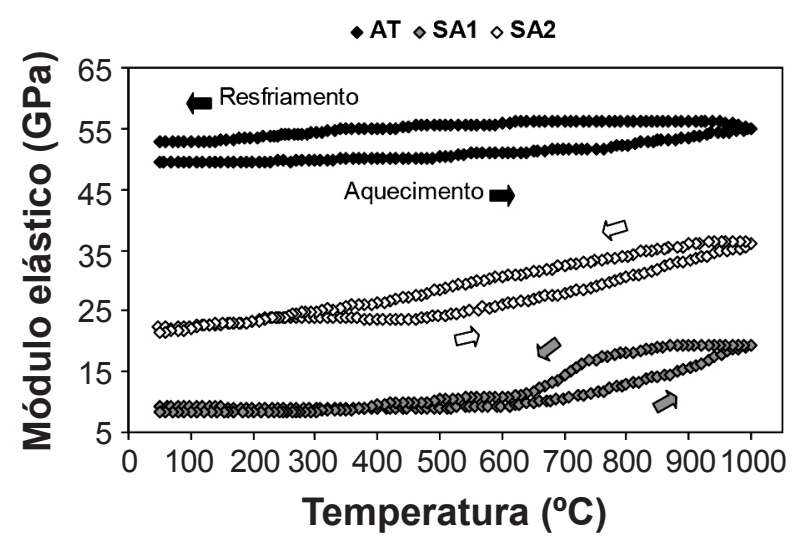

Figura 5: Módulo elástico in situ para o $2^{\circ}$ ciclo térmico dos concretos AT, SA1 e SA2.

[Figure 5: In situ elastic modulus as a function of temperature for AT, SA1 and SA2 castables - second thermal cycle.]

verificada visto que a microestrutura resultante da queima inicial conseguiu absorver as tensões que foram geradas. Isto se deve ao fato dos materiais terem sido submetidos à mesma condição de ensaio utilizada no $1^{\circ}$ ciclo. A Fig. 6 mostra os resultados de resistência à compressão diametral e erosimetria a frio dos concretos AT, SA1 e SA2.

Ao contrário do observado para os materiais AT e SA2, em que a etapa de queima proporcionou um aumento da resistência mecânica, para SA1 houve um decréscimo de $26,9 \%$ em relação ao valor obtido após secagem a $110^{\circ} \mathrm{C}$. Este resultado comprova a severidade do trincamento ocorrido na queima inicial, conforme foi indicado pela curva de módulo elástico em função da temperatura da Fig. 3. Com relação ao desgaste por erosão, mesmo possuindo um teor mais elevado de alumina tabular em sua composição, o concreto SA1 apresentou um volume erodido consideravelmente superior ao de SA2. Isto ocorreu, principalmente, pelo dano ocorrido à microestrutura do material, possibilitando que os agregados fossem mais facilmente desprendidos da matriz. De acordo com os resultados da Fig. 7, o concreto SA1 também obteve um baixo desempenho quanto à solicitação por choque térmico cíclico. Verifica-se que a redução percentual do módulo elástico ao decorrer dos oito ciclos de aquecimento e resfriamento foi muito elevada, superando até mesmo o valor obtido para o concreto a base de alumina 


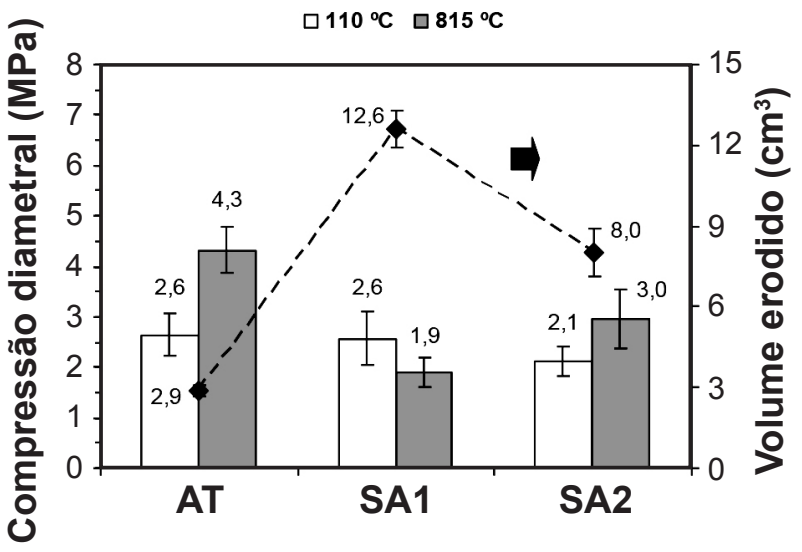

Figura 6: Resultados de compressão diametral (após secagem a $110^{\circ} \mathrm{C}$ e queima a $815^{\circ} \mathrm{C} / 5 \mathrm{~h}$ ) e erosimetria a frio (após queima a $815^{\circ} \mathrm{C} / 5 \mathrm{~h}$ ) dos concretos AT, SA1 e SA2.

[Figure 6: Splitting tensile strength (after drying at $110{ }^{\circ} \mathrm{C}$ and firing at $815^{\circ} \mathrm{C} / 5 \mathrm{~h}$ ) and cold erosion resistance (after firing at $\left.815^{\circ} \mathrm{C} / 5 \mathrm{~h}\right)$ for the castables AT, SA1 and SA2.]
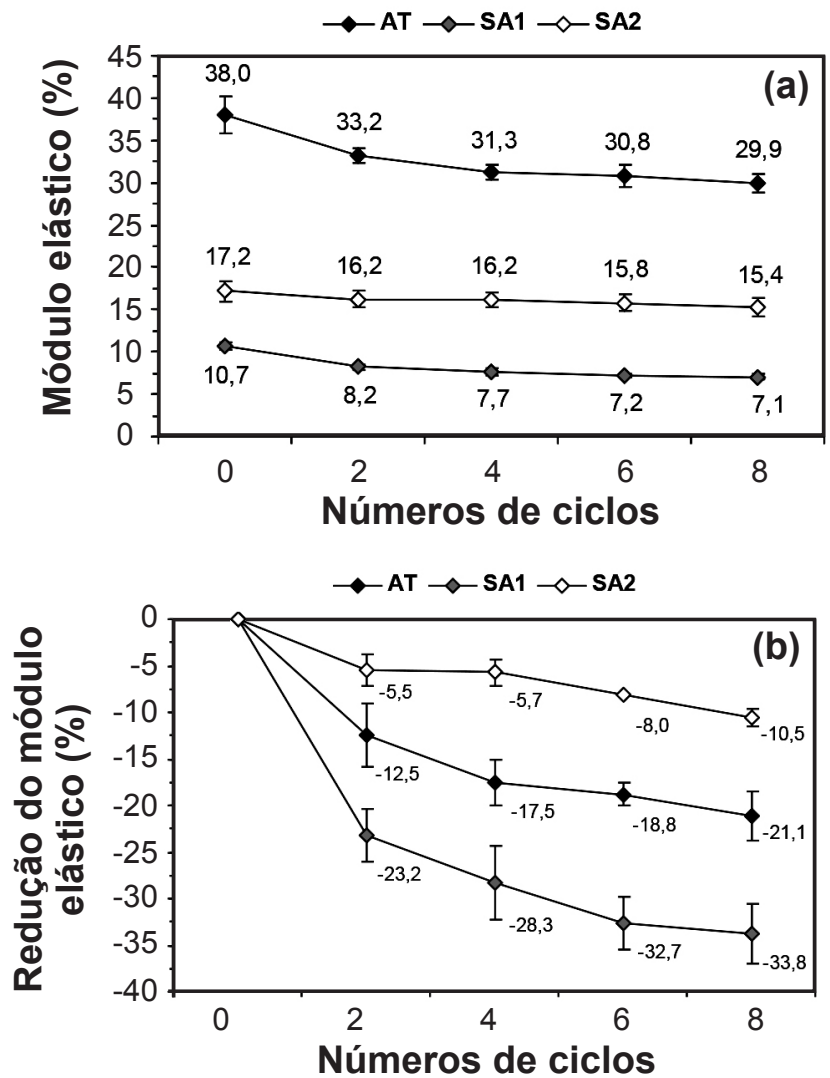

Figura 7: Módulo elástico (a) e sua redução percentual (b) em função do número de ciclos de choque térmico (amostras pré-queimadas a $\left.815^{\circ} \mathrm{C} / 5 \mathrm{~h}\right)$ para os concretos AT, SA1 e SA2 $\left(\Delta \mathrm{T}=790^{\circ} \mathrm{C}\right)$.

[Figure 7: Absolute elastic modulus (a) and the percentage loss (b) for the AT, SA1 and SA2 castables (pre-fired samples at $815^{\circ} \mathrm{C} / 5 \mathrm{~h}$ ) as a function of the thermal shock cycles $\left.\left(\Delta T=790^{\circ} \mathrm{C}\right).\right]$

tabular (AT). A maior tendência à ocorrência de spalling do concreto SA1 seria catastrófica para as condições da aplicação em riser de UFCC, por potencializar o desgaste por erosão e a deposição de carbono. Assim, o concreto SA2 mostrou ser o mais promissor.

Isto fez com que este material fosse selecionado para a incorporação do aditivo sinterizante a base de Al metálico e boro. A Fig. 8 mostra o efeito provocado no perfil do módulo elástico do concreto SA2 durante o $1^{\circ}$ ciclo térmico. Até $700{ }^{\circ} \mathrm{C}$ não houve modificação em relação ao obtido inicialmente para esta composição. Contudo, acima desta temperatura já foi possível verificar a ocorrência de um maior acréscimo do módulo elástico, o qual se estendeu até $1000{ }^{\circ} \mathrm{C}$. Este comportamento foi relacionado à formação de uma fase líquida que contribuiu para a sinterização em baixa temperatura $[10,11]$. No resfriamento, com a solidificação do líquido, o valor de módulo elástico se manteve até $500{ }^{\circ} \mathrm{C}$ e, a partir daí, uma redução gradual do módulo elástico até a temperatura ambiente ocorreu por causa da tensão residual imposta pelo resfriamento, gerando danos à microestrutura do material.

O segundo ciclo (Fig. 9) revelou que à formação de fase líquida durante o primeiro contato com temperatura elevada não ocasionou um efeito deletério, pois até $1000{ }^{\circ} \mathrm{C}$

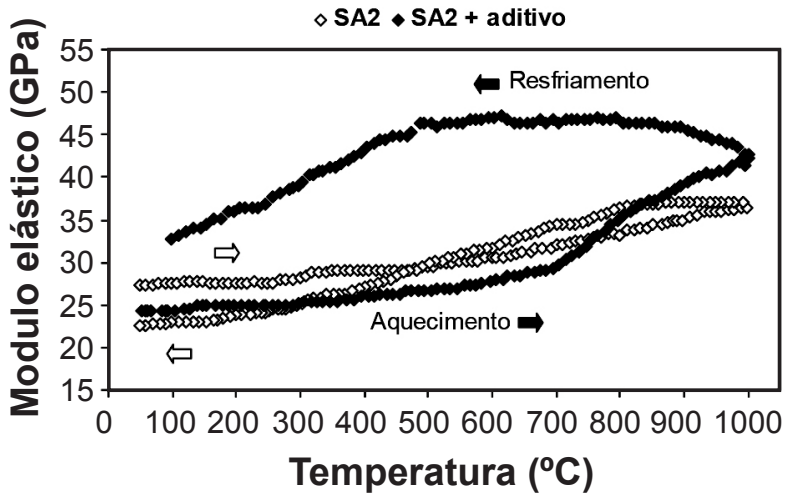

Figura 8: Módulo elástico em função da temperatura para o $1^{\circ}$ ciclo térmico do concreto SA2 contendo aditivo sinterizante.

[Figure 8: In situ elastic modulus as a function of temperature for the SA2 castable with and without sintering additive - first thermal cycle.]

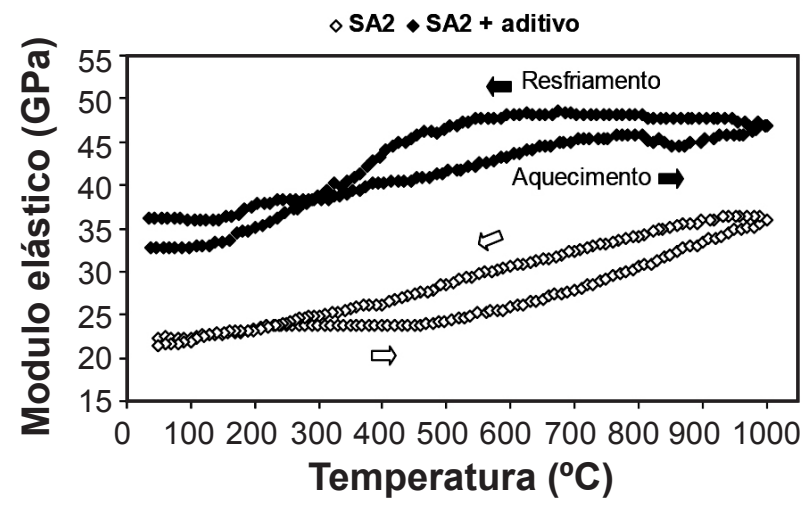

Figura 9: Módulo elástico em função da temperatura para o $2^{\circ}$ ciclo térmico do concreto SA2 contendo aditivo sinterizante.

[Figure 9: In situ elastic modulus as a function of temperature for the SA2 castable with and without sintering additive - second thermal cycle.] 


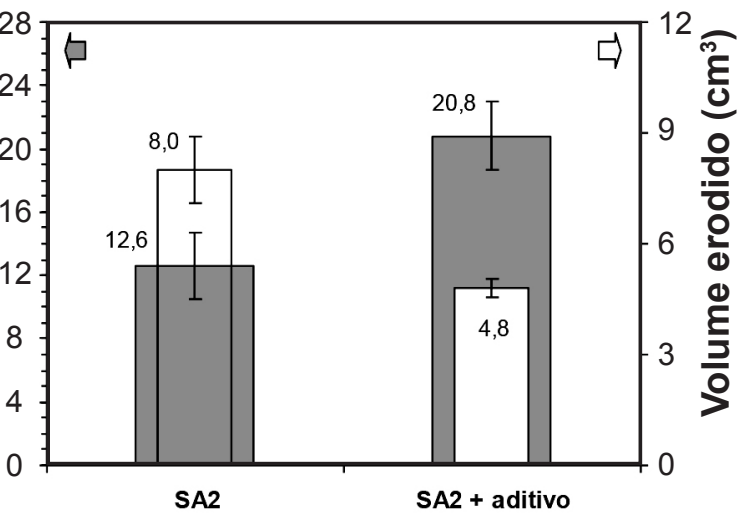

Figura 10: Módulo de ruptura a $815{ }^{\circ} \mathrm{C}$ e erosimetria a frio do concreto SA2 contendo ou não aditivo sinterizante (amostras préqueimadas a $815^{\circ} \mathrm{C} / 5 \mathrm{~h}$ ).

[Figure 10: Modulus of rupture at $815{ }^{\circ} \mathrm{C}$ and cold erosion resistance (pre-fired samples at $815{ }^{\circ} \mathrm{C} / 5 \mathrm{~h}$ ) of the $\mathrm{SA2}$ castable with and without sintering additive.]
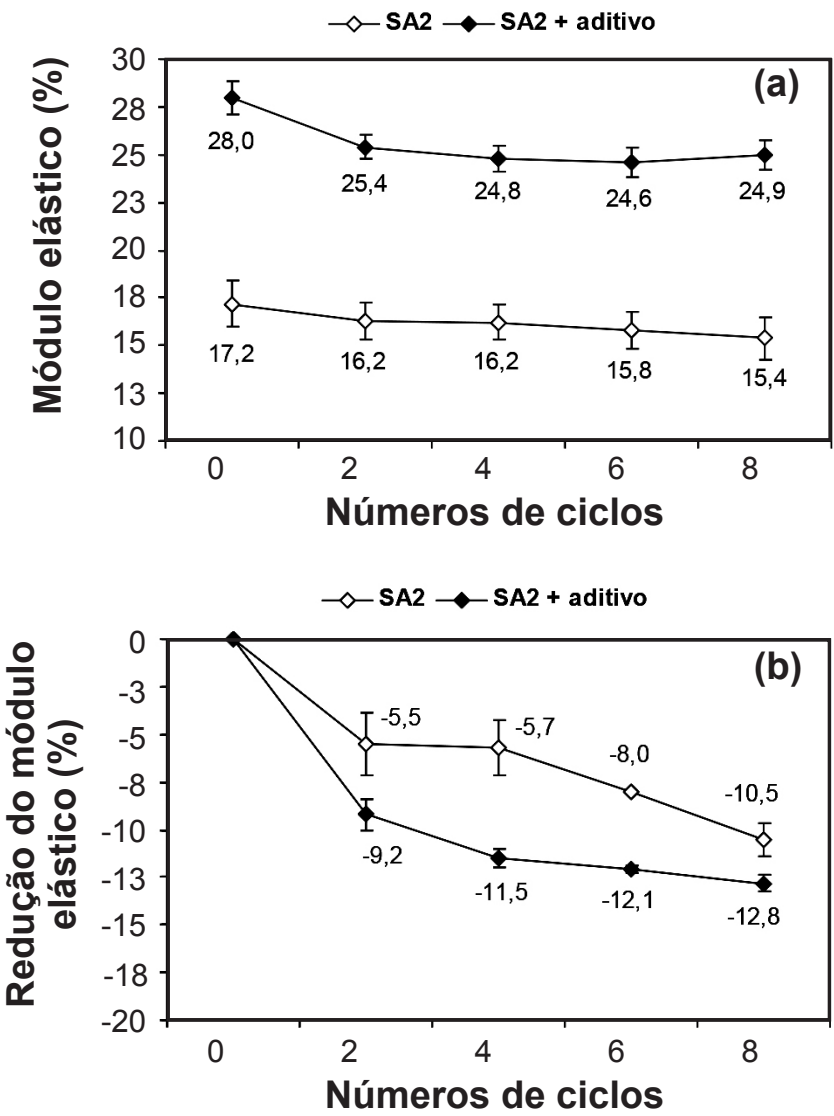

Figura 11: Módulo elástico (a) e sua redução percentual (b) em função do número de ciclos de choque térmico (amostras préqueimadas a $815{ }^{\circ} \mathrm{C} / 5 \mathrm{~h}$ ) do concreto SA2 contendo aditivo sinterizante $\left(\Delta \mathrm{T}=790^{\circ} \mathrm{C}\right)$.

[Figure 11: Absolute elastic modulus (a) and the percentage loss (b) for the SA2 castable with and without sintering additive (prefired samples at $815^{\circ} \mathrm{C} / 5 \mathrm{~h}$ ) as a function of the thermal shock cycles number $\left(\Delta T=790^{\circ} \mathrm{C}\right)$.] não houve tendência de redução do módulo elástico. Com isso, é possível afirmar que as transformações ocorridas no primeiro aquecimento foram permanentes e contribuíram para um significativo acréscimo da resistência mecânica.

Os resultados de módulo de ruptura a $815{ }^{\circ} \mathrm{C}$ e erosimetria a frio da composição SA2+aditivos (Fig. 10) sustentam esta afirmação.

Com a incorporação do aditivo sinterizante o valor médio de volume erodido do concreto SA2 foi reduzido de 8,0 para $4,8 \mathrm{~cm}^{3}$. Como a especificação de desgaste por erosão da indústria petroquímica brasileira para materiais com teor mínimo de $\mathrm{Al}_{2} \mathrm{O}_{3}$ de $80 \%$-p e densidade aparente superior a $2,60 \mathrm{~g} / \mathrm{cm}^{3}$ é de até $6 \mathrm{~cm}^{3}$ após queima a 815 ${ }^{\circ} \mathrm{C}$ [16], pode-se afirmar que o resultado obtido foi muito promissor. A Fig. 11 apresenta o efeito da incorporação do aditivo sinterizante na resistência ao choque térmico.

Um baixo nível de redução percentual do módulo elástico ao final dos ciclos de choque térmico foi mantido. Contudo, houve um acréscimo do valor absoluto de módulo elástico final para a composição contendo o aditivo de sinterização Este último efeito foi muito significativo e indica uma melhoria na resistência ao choque térmico do material. Desta forma, pela incorporação do aditivo sinterizante, foi possível minimizar o desgaste por erosão e aumentar a resistência ao choque térmico do concreto nanoligado.

\section{CONCLUSÕES}

O estudo realizado para o desenvolvimento de um concreto a base de sílica fundida de elevada resistência à erosão mostrou que a utilização do agregado de alumina tabular gera, neste sistema, um grande mismatch de expansão, ocasionando um elevado dano à microestrutura do material. Como resultado, detectou-se um aumento do desgaste por erosão e menor resistência ao choque térmico. Este comportamento mostra a existência de uma restrição quanto à possibilidade de combinações entre os diferentes agregados. Uma solução adequada foi obtida adicionando-se a alumina somente em frações mais finas do agregado e da matriz. Nesta composição de densidade aparente de apenas $2,16 \mathrm{~g} / \mathrm{cm}^{3}$, a incorporação de um aditivo sinterizante constituído por uma combinação entre Al metálico e um composto a base de boro, permitiu a obtenção de um valor médio de desgaste por erosão de $4,8 \mathrm{~cm}^{3}$, após queima a $815^{\circ} \mathrm{C}$. Em comparação com os $6 \mathrm{~cm}^{3}$ especificados pela indústria petroquímica brasileira para produtos refratários de alta alumina com densidade aparente superior a $2,60 \mathrm{~g} / \mathrm{cm}^{3}$, pode-se afirmar que o resultado obtido foi muito promissor. Com isso, o concreto desenvolvido mostrou ser uma excelente opção para aplicação em riser de UFCC.

\section{AGRADECIMENTOS}

Os autores agradecem ao CNPq e a Petrobrás pelo apoio na realização deste trabalho. 


\section{REFERÊNCIAS}

[1] J. R. Peterson, "Refractory quality standards for oil refining applications", Refract. Appl. News 7, 4 (2002) 2427.

[2] M. O'Driscoll, "Refractories in petroleum refining", Ind. Minerals 403 (2001) 29-33.

[3] L. R. M. Bittencourt, M. R. Fernandes, S. L. C. da Silva, B. M. Rulff, "The effect of coke deposition on the mechanical and thermomechanical properties of dense and insulating castable used in FCC units", Unitecr'03 (2003) 549-552.

[4] V. A. A. dos Santos, F. A. O. Valenzuela, J. B. Gallo, V. C. Pandolfelli, "Fundamentos e testes preliminares de resistência à erosão em concretos refratários", Cerâmica 52, 323 (2006) 128-135.

[5] N. Tessier-Doyen, J. C. Glandus, M. Huger, "Untypical Young's modulus evolution of model refractories at high temperature", J. Eur. Ceram. Soc. 26, 3 (2006) 289-295.

[6] G. Briche, N. Tessier-Doyen, M. Huger, T. Chotard, "Investigation of the damage behaviour of refractory model materials at high temperature by combined pulse echography and acoustic emission techniques", J. Eur. Ceram. Soc. 28, 15 (2008) 2835-2843.

[7] S. Wiederhorn, "Erosion of castable refractories", Refract. Appl. News 2, 1 (1997) 2-6.

[8] M. R. Ismael, R. D. Anjos, R. Salomão, V. C. Pandolfelli, "Colloidal silica as a nanostructured binder for refractory castables", Refract. Appl. News 11, 4 (2006) 16-20.

[9] M. A. L. Braulio, C. Tontrup, J. Medeiros, V. C. Pandolfelli, "Colloidal alumina as a novel castable bonding system”, Ref. W. Forum 3, 3 (2011) 135-141.

[10] M. A. L. Braulio, G. G. Morbioli, J. Medeiros, J. B. Gallo, V. C. Pandolfelli "Nano-bonded wide temperature range designed refractory castables", J. Am. Ceram. Soc 95, 3 (2012) 1100-1104.

[11] E. Prestes, "High-performance nano-bonded castables for petrochemical applications", Ref. W. Forum 4, 3 (2012) 111-116.

[12] I. R. Oliveira, A. R. Studart, R. G. Pileggi, V. C. Pandolfelli, Dispersão e empacotamento de partículas - princípios e aplicações em processamento cerâmico, Fazendo Arte Ed., S. Paulo, Brasil (2000) p. 119.

[13] M. R. Ismael, F. T. Ramal JR, V. C. Pandolfelli, "Sol de sílica como agente ligante para concretos refratários", Cerâmica 52, 321 (2006) 82-87.

[14] R. G. Pileggi, V. C. Pandolfelli, A. E. Paiva, J. Gallo, "Novel rheometer for refractory castables", Am. Ceram. Soc. Bull. 79, 1 (2000) 54-58.

[15] H. Baudson, F. Debucquoy, M. Huger, C. Gault, M. Rigaud, "Ultrasonic measurement of Young's modulus $\mathrm{MgO} / \mathrm{C}$ refractories at high temperature", J. Eur. Ceram. Soc. 19, 10 (1999) 1895-1901.

[16] F. A. S. Serra, E. Prestes, J. Medeiros, J. L. B. C. Veiga, V. C. Pandolfelli, "Seleção de concretos refratários densos antierosivos para unidades de craqueamento catalítico fluidizado de petróleo", Cerâmica 58, 345 (2012) 8-13.

[17] D. M. Liu, E. J. Winn, "Microstresses in particulatereinforced brittle composites", J. Mater. Sci. 36, 14 (2001) 3487-3495.

[18] A. A. Fahmy, A. N. Ragai, "Thermal-expansion behaviour of 2-phase solids", J. Appl Phys. 41, 13 (1970) 5108-5111.

[19] D. J. Green, "Stress-induced microcracking at secondphase inclusions", J. Am. Ceram. Soc. 64, 3 (1981) 138-141. [20] Y. Joliff, J. Absi, J. C. Glandus, M. Huger, N. Tessier-Doyen, "Experimental and numerical study of the thermomechanical behaviour of refractory model materials", J. Eur. Ceram. Soc. 27, 2-3 (2007) 1513-1520.

[21] T. Chotard, J. Soro, H. Lemercier, M. Huger, C. Gault, "High temperature characterisation of cordierite-mullite refractory by ultrasonic means", J. Eur. Ceram. Soc. 28, 11 (2008) 2129-2135.

(Rec.03/12/2012, Ac.03/04/2013) 